\title{
Estrategias de evaluación mediadas por las tecnologías de la información y comunicación (TIC): Una revisión de bibliografía
}

\author{
Evaluation Strategies Mediated by ICT: A Literature Review
}

\section{Estratégias de Avaliação Mediadas por TIC: Uma revisão da literatura}

Julián Dávila-Gilede

Universidad Militar Nueva Granada

Ingeniería Industrial

Bogotá, Colombia

julian.davila@unimilitar.edu.co

https://orcid.org/0000-0002-4660-3806

Recibido • Received • Recebido: 12 / 07 / 2019

Corregido • Revised • Revisado: 18 / 03 / 2021

Aceptado • Accepted • Aprovado: 06 / 04 / 2021

\begin{abstract}
Resumen: El presente artículo tiene como propósito develar las estrategias de evaluación mediadas por las tecnologías de la información y comunicación (TIC), de acuerdo con una revisión sistemática de bibliografía configurativa y agregativa, que hace uso de teoría fundamentada para el análisis de datos. Para el caso particular, se tuvieron en cuenta las estrategias, usos, tipos de evaluación y herramientas que se han utilizado en el periodo 2010-2016 para mediar o para apoyar los procesos evaluativos. Como evidencia principal, es notorio que el uso de TIC en los procesos de evaluación ha tomado fuerza gradualmente y su integración tiene diversos propósitos. Se concluye que es necesario tener en cuenta la estrategía, el tipo de evaluación que se quiere favorecer, las TIC o tecnología disponible y ambiente de aprendizaje donde se desarrolla el proceso educativo.
\end{abstract}

Palabras claves: Evaluación educativa; tecnologías de la información; tecnología educativa; revisión de la bibliografía. 
http://doi.org/10.15359/ree.25-2.16

http://www.una.ac.cr/educare

educare@una.ac.cr

\begin{abstract}
This article aims to present evaluation strategies through information and communication technologies (ICT), using a configurative and aggregative systematic literature review, employing grounded theory for data analysis. For this particular case, strategies, uses, types of evaluations, and tools, used in recent years (2010-2016) to mediate or support evaluation processes were taken into account. As main evidence, it is well known that the use of ICT in evaluation processes has gradually strengthened, and its inclusion has several purposes. It is concluded that it is necessary to bear in mind the strategy, type of evaluation required to favor, ICT or technology available, and the learning environment for the education process.
\end{abstract}

Keywords: Educational assessment; information technology; technology education; literature reviews.

Resumo: O objetivo deste artigo é revelar as estratégias de avaliação mediadas pelas Tecnologias da informação e comunicação (TIC), de acordo com uma revisão sistemática da literatura configurativa e agregativa, valendo-se da teoria fundamentada para a análise dos dados. Para o caso particular, foram consideradas as estratégias, usos, tipos de avaliação e ferramentas utilizadas nos últimos anos (2010-2016) para mediar ou apoiar os processos de avaliação. Como evidência principal, é de conhecimento que o uso das TIC nos processos de avaliação ganhou força gradualmente e sua integração tem várias mudanças. Conclui-se que é necessário levar em consideração a estratégia, o tipo de avaliação a ser favorecida, as TIC ou a tecnologia disponível e o ambiente de aprendizagem em que o processo educacional é desenvolvido.

Palavras-chave: Avaliação educacional; tecnologia da informação; tecnologia educacional; revisão de literatura.

\title{
Introducción
}

La evaluación es tal vez uno de los componentes educativos que más requiere atención, un desafio que, aunque ha tenido avances, aún necesita esfuerzos que respondan a los cambios y transformaciones que están surgiendo en las prácticas educativas y de aula. Por tal razón, se hace necesario repensar el sistema de evaluación como un sistema que reconozca los diferentes tipos y niveles de aprendizaje que se pueden reportar antes, durante y después de un proceso educativo formal e informal. Un sistema que no se limite a preguntar contenidos, fórmulas y resultados, sino un sistema que sea capaz de identificar, realimentar y valorar procedimientos, avances y resultados diferentes, dependiendo de lo que se pretenda evaluar y del sujeto evaluado; por esta razón las tecnologías son un instrumento mediador importante que aportará en la solución del presente desafío.

Ausubel (1968) mencionaba y afirmaba que el elemento más importante que influía en el aprendizaje del estudiantado, era lo que el alumnado ya sabía, y que por lo tanto era deber del profesorado averiguarlo y partir de allí para planear lo que se debía enseñar (Ausubel, 1968). Lo anterior tiene mucha validez y suficiente evidencia para demostrarlo, pero también es cierto que 
con el trascurrir del tiempo se ha evidenciado que el estudiantado no aprende lo que se le enseña, en varios casos los resultados de aprendizaje tienen poca relación con su pretensión inicial (Wiliam, 2011). Por eso fue necesario ampliar el significado reducido de evaluación o su concepción inicial, que únicamente se enfocaba en la eficacia de las secuencias de actividades propuestas.

En este sentido y continuando con la idea anterior, la evaluación ha estado en continuo cambio, orientada principalmente por los contextos de enseñanza-aprendizaje (Izquierdo, 2008) y por diversas preocupaciones que suscitan los procesos y estrategias que a través del tiempo se han construido, tales como el desarrollo específico y significativo de una evaluación cognitiva y transitoria, donde los instrumentos tylerianos permiten medir y cuantificar los objetivos de la evaluación educativa. Pero también, en otros contextos en donde ha respondido a procesos de evaluación enfocados en la metacognición y evaluaciones de tipo social (Bernard et al., 2014; Dochy et al., 2002).

En concordancia con lo anterior, se puede afirmar que existen diversas concepciones evaluativas, estructuradas y fundamentadas desde la perspectiva que lo aborde, como puede ser lo pedagógico, didáctico, metodológico, instrumental o estratégico, y únicamente por citar algunos ejemplos en los que se ha reflexionado el fenómeno de la evaluación en las últimas decadas, pueden ser el aprendizaje basado en problemas, aprendizaje basado en proyectos, aprendizaje basado en juegos, la evaluación de portafolios y el análisis y solución de casos, entre otros (Rust, 2002). Para algunas instituciones y sus currículos, estas estrategias pueden ser nuevas; su implementación lograría una mejora en los procesos de evaluación, si se cumplen las siguientes premisas: a) Lograr que el estudiantado se sienta activo y parte del proceso de evaluación; b) entender que la autoevaluación hace parte de su proceso de mejora; c) poder procesar y entender los resultados como mejora de evaluación, no solo para el estudiantado, sino para el personal docente (Bordas Alsinay Cabrera Rodríguez, 2001).

Con el fin de renovar y transformar algunas de las estrategias que se vienen implementando en los procesos de enseñanza y aprendizaje y, por supuesto, en la evaluación, se observan las TIC como mediadoras del proceso, en donde es posible encontrar que la aplicación de estas mismas se hace posible a partir del interés docente, con la utilización de estrategias como las nombradas anteriormente y el fomento del cambio en las prácticas de enseñanza-aprendizaje y evaluación (Muñoz, 2008). La comunicación y la información serán de suma importancia para crear entornos de aprendizaje en los cuales la interactividad, los canales de información y la comunicación activa son parte del proceso educativo (García-Valcárcel-Muñoz-Repiso et al., 2014).

Como se indicó anteriormente, la tecnología se ha venido incorporando paulatinamente a los procesos de enseñanza-aprendizaje, e intrínsecamente en la evaluación, contribuyendo con la generación de dinámicas actuales y en algunos casos disruptivas o inimaginables en 
http://doi.org/10.15359/ree.25-2.16

http://www.una.ac.cr/educare

educare@una.ac.cr

educación, por tal razón su uso e integración se percibe como necesaria y benefactora. Pero, es necesario tener en cuenta que no solo es importante aplicar la tecnología como instrumento de acompañamiento en cualquier proceso de enseñanza, sino es importante definir una estrecha relación entre lo que se enseña y lo que se quiere evaluar, además de una pertinente y coherente selección de la tecnología en relación con la metodología y estrategia utilizada (Esteve Mon y Gisbert Cervera, 2011).

A este tipo de estrategias se le conoce como tecnología basada en evaluación, su intención es de alto alcance, ya que es un método útil para el desarrollo y administración de evaluaciones, puntajes, evaluaciones en línea, análisis de datos y, finalmente, un punto importante en la evaluación y retroalimentación (van Niekerk et al., 2010).

Las tecnologías utilizadas en la evaluación deben considerarse como herramientas mediadoras que contribuyen y potencian las estrategias, así mismo, deben reflejar siempre la mejora delos procesos deenseñanzayaprendizaje (Hersh,2014).Estos procesos conla integración de la tecnología deben realizarse en contexto, ya sea social o tecnológico, para desarrollar una correcta aplicación y tener un control de su efectividad en la actividad correspondiente (García Laborda et al., 2015). A parte del contexto social y tecnológico, también será relevante tener en cuenta la teoría de evaluación sobre la cual se está trabajando la mencionada estrategia, para identificar el fin, tipo de evaluación y lo que se pretende evaluar; con estos parámetros, será sencillo identificar una herramienta TIC o una tecnología que medie el proceso, teniendo en cuenta el conocimiento conceptual y procedimental de esta (Pásztor et al., 2015).

A partir de lo expuesto anteriormente de manera sucinta, se evidencia que la evaluación mediada por TIC o la tecnología basada en evaluación aún es un campo en desarrollo, tal vez prematuro, pero sobre el cual se han realizado diferentes esfuerzos necesarios de compilar y sistematizar, con el propósito de ver los avances y poder determinar posibles vacíos. Por tal razón la presente revisión se orientó a responder la siguiente pregunta: ¿Qué estrategias de evaluación se han mediado por TIC en los procesos de enseñanza-aprendizaje en los últimos seis años 2010-2016?

\section{Método}

\section{Tipo de estudio}

De acuerdo con la propuesta de Fink (2013), se propuso una revisión sistemática a la bibliografía (RSB), concibiéndola como un método explícito y reproducible que permite identificar, evaluar y sistematizar el cuerpo existente y completo de determinado campo o tema. El procedimiento se realizó siguiendo los siete pasos propuestos: Pregunta de investigación (PI), 
selección de bases de datos (BD), elegir términos de búsqueda (TB), aplicar filtros y límites (FL), aplicar la calidad metodológica (CM), sintetizar los resultados (SR) y consolidar y producir la revisión (CPR).

La fase de SR se apoyó en lo propuesto por Esteve Mon y Gisbert Cervera (2011), cuando se refiere a revisión configurativa, y la fase de PI y SR se fundamentó en Gough et al. (2012) cuando se refiere a revisión agregativa. En el caso de revisión configurativa, se pretenden identificar conceptos que busquen la heterogeneidad a través de patrones repetitivos que se puedan sintetizarse en categorías o conceptos para ser analizados. Por su parte, la revisión agregativa se encarga de buscar evidencias para mostrar las decisiones obtenidas en los diferentes casos; a menudo estas revisiones están basadas en la búsqueda de conceptos y métodos prestablecidos para generar una revisión exhaustiva.

Para este caso en particular y teniendo en cuenta lo anterior, se propuso una PI amplia que respondiera al carácter agregativo, con el fin de identificar un cúmulo de bibliografía importante que permitiera responder a la pregunta planteada desde diferentes perspectivas y estudios. En un segundo momento SR, fue necesario establecer categorías de análisis de datos, las cuales fueron a priori y emergentes, por cuyo medio se logró consolidar e identificar ciertos elementos que respondián a la pregunta propuesta coherentemente con el tipo de revisión configurativa.

Por lo tanto, la presente revisión hace una descripción a través de un análisis de cada categoría por separado, para luego triangular las categorías con el propósito de responder a la pregunta que orienta la presente investigación.

\section{Procedimiento}

De acuerdo con el modelo seleccionado para realizar la revisión sistemática, se siguieron cada una de las fases del procedimiento, las cuales se detallan en la Tabla 1.

\section{Muestra}

Los resultados iniciales de la búsqueda de artículos en la fase FL estuvo compuesta de la siguiente manera: Science Direct 155, Scopus 36 y WOS 65 artículos respectivamente. Para un total de 256 artículos como población total. Fue necesario eliminar duplicados y, por esta razón, quedaron 242 documentos. Seguido al proceso inicial, se realizó un proceso de abstrating (lectura de título, abstract y keywords), por parte de dos investigadores que hacían lectura y observación de manera separada. El fin de esa lectura tenía fue identificar la muestra definitiva de artículos que serían analizados de acuerdo con la afinidad y cohesión respecto a la PI. 
http://doi.org/10.15359/ree.25-2.16

http://www.una.ac.cr/educare

educare@una.ac.cr

Tabla 1: Fases del método y descripción del procedimiento

\section{Fases Descripción del procedimiento realizado}

PI Se determinó la pregunta de investigación que orientaba la revisión: ¿Qué estrategias de evaluación se han mediado por TIC en los procesos de enseñanza-aprendizaje en los últimos seis años 2010-2016?

BD Se seleccionaron las bases de datos: WOS (Web of Science), Scopus y Science Direct.

TB Términos de búsqueda: Technology Assessment y ICT Assessment.

Ecuación de búsqueda: ("Technology Assessment" OR "ICT Assessment") NOT Medicine NOT Health

En WOS (Web of Science), se limitó a que la ecuación estuviese en el título.

En Science Direct y Scopus se limitó a que la ecuación estuviese en el título, resumen y palabras clave.

FL Filtros por área de conocimiento: Education Educational Research, Multidisciplinary Sciences, Education Scientific Disciplines and Engineering Multidisciplinary y Social science. Se excluyó todas las áreas relacionadas con Ciencias de la salud.

Idiomas: Inglés y español

Fecha de publicación: 2010-2016

Tipo de documento: Artículo de revisión y de investigación

CM Se procedió a revisar cada uno de los artículos a través de un proceso de abstrating (lectura de título, abstract y keywords), con el propósito de determinar la muestra definitiva de artículos que serían analizados a profundidad.

Este procedimiento se realizó a través de un coeficiente de Kappa de Cohen (Cohen, 1968), en el que el equipo investigador revisaba los elementos antes mencionados de cada uno de los artículos y se iba construyendo una matriz que permitió obtener la muestra definitiva de artículos a analizar.

SR Se conformó una unidad hermenéutica en el software ATLAS.ti, con la muestra definitiva, en donde cada artículo se constituye como un documento primario, se crearon las categorías a priori y se realizó la respectiva red semántica.

Luego se hizo un conteo de palabras para identificar y contrastar posibles categorías de análisis emergentes y se procedió con la lectura a profundidad.

Se realizó inicialmente una codificación abierta de cada documento, y luego se procedió a realizar una codificación axial, que permitió establecer relaciones entre las categorías y sbucategorías. Por último, se realizó un proceso de codificación selectiva, la cual permitió responder de manera coherente a la pregunta de investigación.

CPR Despúes de haber terminado el análisis de todos los documentos, se comenzó con la elaboración del artículo de revisión, siguiendo el formato IMRaD (Introducción, métodos, resultados y discusión), el cual permitía tener una estructura organizada y común (Wu, 2011).

Nota: Elaboración propia. 
http://doi.org/10.15359/ree.25-2.16

Lo anterior se hizo a través de un índice de Kappa de Cohen (Cohen, 1968), en el cual se diligenció una matriz en la cual se registraba, si el artículo debería ser incluido o no. En este proceso, el resultado de la lectura comparada indicó un coeficiente de 0,94, con una desviación estándar de 0,025 y con un índice de concordancia entre ambos observadores entre el 0,895 y el 0,993 , lo cual evidencia una fuerza de concordancia catalogada como muy buena. De esta manera, la muestra definitiva estuvo compuesta por 52 artículos que fueron leídos a profundidad y analizados en el sotfware ATLAS.ti con la siguiente distribución: Science Direct 19, Scopus 25 y en WOS 8.

\section{Análisis de datos}

Teniendo un corpus documental identificado de 52 artículos, se procedió a constituir una unidad hermenéutica en el software ATLAS.ti. Allí fueron registrados cada uno de los artículos como documentos primarios, se crearon las categorías y subcategorías de análisis a priori que fueron constituidas a partir de la pregunta de investigación. Luego se realizó un conteo de palabras, con el fin de identificar posibles categorías y subcategorías emergentes y contrastar las categorías que ya estaban definidas.

Este proceso fue relevante, permitió identificar repetición de palabras que podían dar un panorama inicial de lo que se encontraría en los datos, y establecer algunas relaciones iniciales entre categorías y subcategorías que luego podrían ser analizadas. Las categorías que siempre estuvieron presentes fueron las que aparecen en la Tabla 2.

Tabla 2: Categorías de análisis y su descripción

\begin{tabular}{ll} 
Categoría & \multicolumn{1}{c}{ Descripción } \\
\hline Evaluación & $\begin{array}{l}\text { Entendida como el método de evaluación aplicado en las diferentes investigaciones, Los más } \\
\text { comunes fueron: autoevaluación, evaluación por pares, evaluación on-line, entre otros. }\end{array}$ \\
Estrategias & $\begin{array}{l}\text { Metodología y proceso que se utilizó para implementar el método de evaluación. Hace alusión al } \\
\text { cómo se impletó, a la didáctica o a la técnica. }\end{array}$ \\
TIC & $\begin{array}{l}\text { Tecnología utilizada para desarrollar o apoyar las metodologías en cada investigación, entendiendo que } \\
\text { cada una de ellas tiene una integración en diferentes niveles de acuerdo con la apropiación y contexto. }\end{array}$
\end{tabular}

Nota: Elaboración propia. 
http://doi.org/10.15359/ree.25-2.16

http://www.una.ac.cr/educare

educare@una.ac.cr

Se propuso aplicar teoría fundamentada para el análisis de los datos, no con el fin de construir teoría, pero sí con el propósito de tener una suficiente claridad en los procedimientos de análisis y realizar una interpretación de datos sistemática. En este caso, se observa que Strauss y Corbin (2002) proponen partir de la codificación abierta. Entendiendo esta como el proceso en donde se aborda cada texto con el fin de realizar un estudio a profundidad que permita comprender el proceso a la luz de las categorías y subcategorías existentes, pero también identificando posibles categorías que emerjan de los datos. Aquí también se hizo uso de los memos, que ayudaban a comprender la razón de una codificación y posibles comprensiones personales que luego serían cotejadas por los investigadores. En esta primera fase, se tuvo un listado de nuevas subcategorías que no se tenían antes y que enriquecían el análisis.

La siguiente fase, continuando con la propuesta de Strauss y Corbin (2002), fue realizar la codificación axial, en donde se establecieron relaciones entre las categorías y subcategorias, para lo cual se construyeron redes semánticas por cada categoría y una red semántica general que permitiera identificar las relaciones entre categorias y subcategorías de toda la investigación, tal como se evidencia en la Figura 1.

Figura 1: Red semántica, proceso de análisis y codificación axial

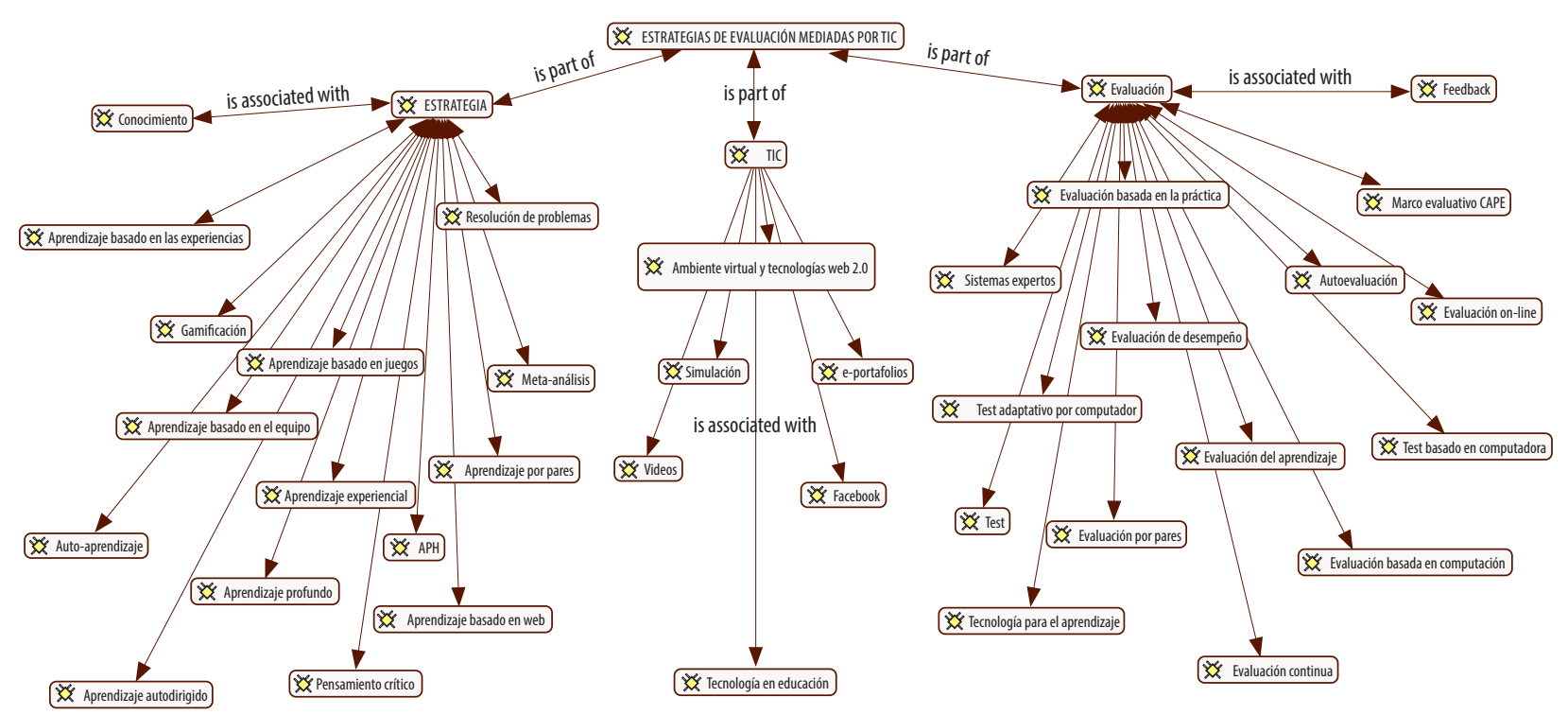

Nota: Elaboración propia.

En las dos fases anteriores, codificación abierta y axial, el proceso fue de comparación constante; cada investigador realizaba la codificación de los documentos de manera independiente hasta tener una saturación de los datos a partir de la precisión (San Martín Cantero, 2014). 
http://doi.org/10.15359/ree.25-2.16 http://www.una.ac.cr/educare educare@una.ac.cr

Por último, se realizó un proceso de codificación selectiva, la cual permitió responder, de manera coherente, a la pregunta de investigación. Este proceso viene muy relacionado con la codificación axial y fue sencillo, al tener en cuenta la red semántica y lo que permite ATLAS.ti. A partir de la red semántica, se comenzaron a construir esas pequeñas frases y narraciones que permitían responder a la pregunta de investigación planteada.

\section{Resultados}

De acuerdo con el proceso de análisis de datos realizado anteriormente, se presentaron los resultados por cada una de las categorías, y luego se hizo una triangulación entre las categorías. Esto permite responder a la pregunta de investigación en el apartado de discusión. En la Figura 2, se evidencian las categorías y subcategorías con sus respectivas frecuencias en la codificación en ATLAS.ti.

Figura 2: Frecuencia de categorías y subcategorías de análisis

\begin{tabular}{|c|c|c|c|c|c|}
\hline Nombre & Fundamentado & Densidad & Autor & Creado & Modifica... \\
\hline e-portafolios & 20 & 0 & Super & $01 / 11 / 20 \ldots$ & 08/11/20... \\
\hline Simulación & 19 & 0 & Super & $02 / 11 / 20 \ldots$ & 09/11/20... \\
\hline Feedback & $\square$ & 0 & Super & $24 / 10 / 20 \ldots$ & 08/11/20... \\
\hline Facebook & $\square$ & 0 & Super & 19/10/20... & 08/11/20... \\
\hline Evaluación por pares & $\mathbf{r}$ & 0 & Super & 19/10/20... & 08/11/20... \\
\hline Tecnología en educación & 口 & 0 & Super & $30 / 10 / 20 \ldots$ & 07/11/20... \\
\hline Ambiente virtual y tecnologías web 2.0 & $\square$ & 0 & Super & 19/10/20... & 07/11/20... \\
\hline Resolución de problemas & - & 0 & Super & $02 / 11 / 20 \ldots$ & 09/11/20... \\
\hline Aprendizaje basado en juegos & 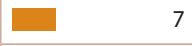 & 0 & Super & $06 / 11 / 20 \ldots$ & 06/11/20... \\
\hline Sistemas expertos & 口 & 0 & Super & $02 / 11 / 20 \ldots$ & 09/11/20... \\
\hline Evaluación & - & 0 & Super & $01 / 11 / 20 \ldots$ & 08/11/20... \\
\hline Autoevaluación & - & 0 & Super & 07/11/20... & 07/11/20... \\
\hline Aprendizaje basado en web & $\mathbf{a}$ & 0 & Super & 19/10/20... & 19/10/20... \\
\hline Evaluación para el aprendizaje & $\square$ & 0 & Super & $31 / 10 / 20 \ldots$ & $31 / 10 / 20 \ldots$ \\
\hline Evaluación on-line & $\square$ & 0 & Super & $01 / 11 / 20 \ldots$ & 07/11/20... \\
\hline Marco evaluativo CAPE & $\square$ & 0 & Super & 03/11/20... & 03/11/20... \\
\hline Aprendizaje basado en experiencias & 口 & 0 & Super & 06/11/20... & 08/11/20... \\
\hline Pensamiento crítico & 口 & 0 & Super & $02 / 11 / 20 \ldots$ & 06/11/20... \\
\hline Aprendizaje por pares & 口 & 0 & Super & 03/11/20... & 03/11/20... \\
\hline Sistemas expertos & 口 & 0 & Super & 03/11/20... & 03/11/20... \\
\hline
\end{tabular}

Nota: Elaboración propia.

\section{Evaluación}

La práctica evaluativa está fundamentada en la intencionalidad formativa de cualquier sujeto que se encuentre en un proceso de enseñanza-aprendizaje, en donde es posible 
http://doi.org/10.15359/ree.25-2.16

http://www.una.ac.cr/educare

educare@una.ac.cr

realizar una validación de resultados, los cuales son obtenidos de manera sistemática en su implementación a partir de la conjunción de una estrategia de implementación y de manera opcional la mediación de una TIC congruente con el proceso. Partiendo desde la idea anterior, es posible encontrar diferentes tipos de evaluación, dependiendo del contexto en el que se presente y de la población en la que se aplique. Para los entornos de aprendizaje presencial es posible encontrar la autoevaluación (Barry, 2012), la evaluación por pares y continua (Yuretich y Kanner, 2015); así mismo, con el avance de la tecnología educativa y su integración en los proceso de evaluación, se observa la evaluación on-line (Gómez-Escalonilla et al., 2011), la evaluación automática (Sánchez Vera et al., 2013), la evaluación automática escrita (Chapelle y Voss, 2016) y, finalmente, la inmersión total de la tecnología no como herramienta en el proceso, sino como elemento evaluativo propiamente, conocidos como sistemas expertos (Kim et al., 2016).

En el trabajo evaluativo que se desarrolla en ambientes presenciales, uno de los objetivos a los que se desea llegar es que el estudiantado pueda desarrollar un análisis profundo a través de ideas propias y generando interacción entre los diferentes participantes de su entorno. Desde esta perspectiva, el concepto de evaluación por pares y la evaluación continua son tomados en cuenta por parte del personal docente, en donde el trabajo colaborativo y el desarrollo de roles en el estudiantado genera discusión y distinción entre las participaciones de cada grupo, no solamente tratando de fortalecer el trabajo grupal, sino mejorando el análisis del trabajo individual de cada estudiante, así, de esta manera, se cumple con la evaluación continua y activa por la parte docente y estudiantil (Yuretich y Kanner, 2015).

Barry (2012) percibe que la autoevaluación es utilizada como un refuerzo para el trabajo individual del estudiantado por medio de actividades de aprendizaje y un objetivo claro dado por el personal docente en su práctica. Por medio del autodescubrimiento se refuerzan las falencias estudiantiles y se genera un análisis profundo respecto a sus propias capacidades, con el objetivo de que analice su entorno y sea capaz de fortalecer su trabajo en sus propias actividades académicas para alcanzar el objetivo docente propuesto.

Con el avance y el uso de las TIC en procesos de enseñanza-aprendizaje y evaluación, su integración consensuada puede darse de manera exponencial en los procesos educativos. Desde esta perspectiva, se relacionan los ambientes b-learning o mixtos (presencial y virtual), en los cuales los procesos de evaluación pasan de cuestionarios, test, encuestas y trabajos escritos en lapiz y papel a la web 2.0. Como fue dicho por Gómez-Escalonia et al. (2011), ese fenómeno tiene un primer lugar donde la tecnología instrumentaliza el proceso evaluativo y cambia su formato, creando la evaluación electrónica o evaluación on-line. La evaluación on-line se ha encargado de evaluar los aprendizajes del estudiantado por medio de la tradicionalidad, pero existen casos en los cuales el objetivo final de esta evaluación no está inmerso en la suma de conocimientos sino en tomar de la práctica del estudiantado la experiencia que este ha tenido con el contenido y con el análisis que pueda realizar de su trabajo y de la estrategia dada por su docente, enfocadas hacia el desarrollo cognoscitivo del estudiantado y, en muchos casos, hacia la metacognición (Gautreau, 2011). 
http://doi.org/10.15359/ree.25-2.16

Con base en la evaluación on-line, cabe resaltar que esta ha tenido cambios importantes a través del tiempo sobre los contextos b-learning y e-learning, como, por ejemplo, en el uso de elementos multimediales cada vez más avanzados, que parten del compendio de videos, audios e imágenes (Ritchie, 2016) hacia el uso de elementos interactivos personalizados para el aprendizaje de cada estudiante. Esto, sumado al diseño de software especializado en la creación de recursos on-line como en la personalización de los procesos de enseñanza-aprendizaje, ha generado nuevas maneras de evaluar; tal es el caso de la evaluación automática, definida por Sánchez Vera et al. (2013) como una respuesta automática a todas las actividades que pueda presentar o necesitar el personal docente para sus estudiantes. Se entiende que este tipo de sistemas posee un análisis interno de los resultados, tomando mayor importancia en las respuestas equivocadas, las que refuerza en los siguientes intentos del estudiantado; estos registros se envían al personal docente para una correcta retroalimentación para cada estudiante. Otro ejemplo de lo anterior es la evaluación escrita automática, la cual se utiliza en el aprendizaje del lenguaje, donde a partir de los escritos del estudiantado se entrena al software para encontrar errores de ortografía o errores semánticos, esta da un reporte para trabajar sobre los errores. El uso de estas estrategias es variado respecto a lo que se desee evaluar (Chapelle y Voss, 2016).

Por otra parte, es posible encontrar sistemas de evaluación totalmente desarrollados como los sistemas expertos, los cuales se adaptan a las respuestas que los sujetos participantes suelen dar; si se incurre en una respuesta incorrecta, el propio sistema se adapta para reforzar y dar la retroalimentación necesaria para cada tipo de estudiante (Kim et al., 2016). Entre estos sistemas expertos, las estrategias pueden ser diferentes, ya que la intención de estos es probar que el estudiantado puede lograr diferentes desarrollos en otros tipos de actividades y su integración en la evaluación es casi total, como cuando se acompañan de otros elementos ya sean, videos, audios e interacción uno a uno (por ejemplo, un sujeto usuario y un PC).

\section{Estrategias}

Las estrategias de evaluación analizadas en la bibliografía son reportes de investigaciones que se desarrollan con estudiantes de diversos niveles educativos y diferentes temáticas. Es necesario resaltar que muchas de estas estrategias no son explícitas, sino que se deben inferir a partir del método o metodología que cada estudio reporta. Entre estas estrategias es posible encontrar el aprendizaje basado en equipos (Yuretich y Kanner, 2015), aprendizaje basado en web (Shih, 2011), experticia basada en computación (Belland, et al., 2015), el aprendizaje basado en problemas y tareas (Kim et al., 2016), simuladores (Ried, 2010) y tecnologías aplicadas a las estrategias de manera consensuada por el personal docente.

La mayoría de estrategias identificadas proponen un trabajo en grupo o en equipo, lo cual supone un reto mayúsculo para el proceso de evaluación, pues normalmente el producto final fue concebido en colectivo y determinar los aportes de cada estudiante en ese proyecto o en la solución al problema no es nada sencillo (Yuretich y Kanner, 2015). 
http://doi.org/10.15359/ree.25-2.16

http://www.una.ac.cr/educare

educare@una.ac.cr

Partiendo de lo señalado en el párrafo anterior, es posible desarrollar prácticas evaluativas fundamentadas en la experiencia del estudiantado con una situación problémica; tal y como lo nombra Kim et al. (2016), las estrategias como el aprendizaje basado en tareas (ABT), el aprendizaje basado en problemas (PBL) o el aprendizaje basado en proyectos (ABP) parten de un reto cognitivo para el estudiatnado, con el propósito de generar pensamiento crítico y científico en contextos diferentes a la escuela y situándoles en ejemplos reales de empresas o de la vida cotidiana. Por esta razón, el estudiantado requiere de ideas creativas, comunicación para el trabajo en equipo y otras habilidades del siglo XXI que tienen que ver con el uso adecuado de tecnología para resolver, de manera pertinente, la situación que enfrenta en su práctica (Pásztor et al., 2015).

Teniendo en cuenta las estrategias mencionadas anteriormente, recobran importancia los portafolios electrónicos, herramienta útil para el estudiantado y para el personal docente, pues allí se logra evidenciar el desempeño y hacer seguimiento a todo el proceso; son herramientas que acompañan y apoyan el trabajo en grupo $y$, por ende, las estrategias comúnmente presentadas. Los portafolios permiten que el estudiantado presente, de forma organizada, su trabajo y lo que han realizado, evidencia clara del proceso y un elemento muy importante dentro del proceso evaluativo (Gautreau, 2011).

Se hace evidente que el cambio en las prácticas educativas ha fomentado el análisis profundo del estudiantado frente a situaciones de la vida real, donde las TIC cumplen un papel importante al estar inmersas en las estrategias de evaluación.

Otras estrategias encontradas que hacen un uso avanzado de tecnología son la experticia basada en computación, donde, a partir de la combinación entre estrategia y TIC, se logra que, por medio del planteamiento de problemas reales y sus decisiones, el personal docente pueda dar cabida a una realimentación importante, apoyándose en el análisis que hace un software para analizar resultados, en el proceso de evaluación (Belland et al., 2015). De esta misma manera se lleva al estudiantado a ver más allá de un análisis inicial, por medio de la argumentación y el análisis correlacional de la computación.

Por su parte, los simuladores desarrollan importantes cambios en su aplicación y en la evaluación; son parte de una práctica que tiende a desarrollarse por un medio virtual (Ried, 2010). Muchas de estas representaciones virtuales propenden a ser fieles a los entornos que desean representar o a las actividades que se desean simular. Esta intervención TIC debe ir acompañada, siempre, de una estrategia de evaluación que puede ser: test tradicionales tanto pre como post según sea el objetivo, resolución de problemas dentro del contexto, repetidores o entrenadores. Se hace necesario entender que las simulaciones pueden ayudar en el entendimiento de ciertos conceptos de la teoría en la práctica, generan experticia y práctica con el uso de tecnología (Williams et al., 2011). En este mismo sentido, la evolución de las estrategias aplicadas a la evaluación hacia una evaluación automática a través de nuevas tecnologías puede ser entendida desde el proceso analítico jerarquizado (APH en sus siglas en inglés) encargado de encaminar 
http://doi.org/10.15359/ree.25-2.16

la evaluación del estudiantado a través de sus respuestas y el entrenamiento que se le da al software para generar un análisis profundo en las respuestas dadas (Hu, 2016).

Con el avance de la inteligencia artificial, de los sistemas expertos, simuladores y estrategias, también se han desarrollado estrategias para la participación de diferentes contextos y aprendizajes, como es el caso de la gamificación y los juegos serios. Los juegos serios son juegos que involucran un sentido educativo, en donde existe previamente un objetivo de aprendizaje, además, es necesario que el estudiantado permanezca en participación continua (Jagodziński y Wolski, 2015). La gamificación es una estrategia que mantiene las características más importantes del juego, tal como el reto, los puntos y la recompensa para incluirlos en un ambiente educativo y fomentar, de manera diferente, el aprendizaje o el proceso educativo que se desee aplicar, sin olvidar que el proceso debe ser reconocido por el término "divertido" (Figura 3).

Figura 3: Innovación en el uso de las TIC en las estrategias de evaluación actuales, estos usos pueden ser adaptables o prioritarios según su implementación

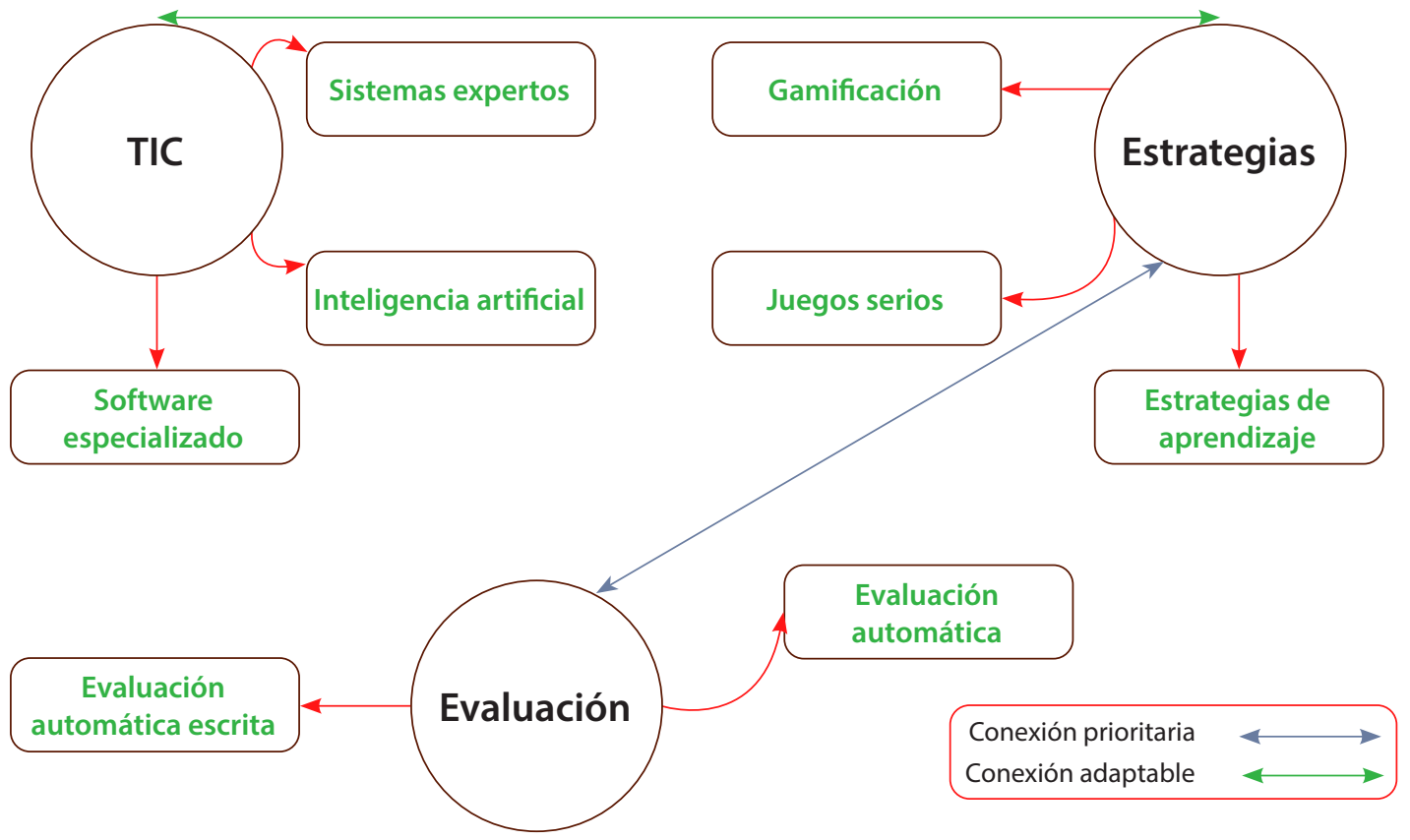

Nota: Elaboración propia.

\section{TIC}

Como se ha podido observar, la integración de las TIC o de la tecnología en los procesos de evaluación está muy acorde con la estrategia planteada por el profesorado, entendiendo, de esta manera que, según las necesidades de aprendizaje del estudiantado, el personal docente ha creado 
http://doi.org/10.15359/ree.25-2.16

http://www.una.ac.cr/educare

educare@una.ac.cr

metodologías en las cuales se trabaja desde el concepto de evaluación combinada con una estrategia de implementación y la mediación TIC. Esta mediación comúnmente puede ser observable en herramientas web 2.0, entendidas como videos, edublogs, redes sociales, test en línea y portafolios, dependiendo de la población objetivo y el contexto de la práctica (Singh, 2013).

Algunos ejemplos de lo anterior pueden observarse en el aprendizaje web, donde se integran y se usan los videos interactivos, que sitúan al estudiantado dentro de un proceso de visualización de video, pero de manera activa, pues este debe visualizar el video y tener interacción a través de preguntas emergentes que se van presentando, con el propósito de corroborar que el video esta siendo visualizado con atención por parte del estudiantado (Ritchie, 2016).

Como lo mencionan Martín-Gámez et al. (2015), herramientas tales como Facebook, Twitter, edublogs toman sentido en el aprendizaje, pues generan una interacción colaborativa entre participantes. Se puede decir que aplicaciones como las redes sociales, al estar conectadas a una gran cantidad de población, pueden ser direccionadas como un centro de construcción de opiniones y desarrollo de análisis, son una poderosa herramienta (Ritchie, 2016). Además, si se lleva a cabo una interacción entre estudiantes-docente, estudiante-contenido y estudianteestudiante, es posible generar un ambiente de aprendizaje virtual mediado por una herramienta TIC como lo puede ser Facebook (Amasha y Alkhalaf, 2015).

Como se ha podido ver en los resultados, existen tecnologías que llevan la interacción y los procesos de enseñanza-aprendizaje y evaluación mucho más allá de lo tradicional. Por ejemplo, la simulación (Maas y Flood, 2011), mostrada en sus diferentes niveles de interacción, puede convertirse en tecnología como apoyo a la evaluación, o en sí misma, tomarse como una estrategia de evaluación clara. De la misma manera, los sistemas expertos, se convierten en estrategias de evaluación y un tipo de evaluación en sí mismos (Pirnay-Dummer et al. (2010). En este punto, la inteligencia artificial y la simulación se complementan para formar sistemas de evaluación que se adaptan a cualquier estrategia o que se constituyen como una estrategia completa en la que se integra la evaluación (Wang, 2014).

\section{Discusión}

A través de la revisión realizada y desde los resultados obtenidos, es posible considerar que las estrategias mediadas o que han utilizado tecnologías se encuentran presentes en cada evaluación realizada, entendiendo que no es posible realizar la evaluación sin una estrategia de implementación clara, en donde las TIC, en ciertos momentos, permiten ser realmente un mediador entre el aprendizaje del estudiantado y lo que el personal docente quiere enseñar. De la misma manera, se encontró que cuando las tecnologías son incluidas sin un sentido educativo definido y sin un contexto, se convierten en un distractor del objetivo final y, por ende, se interponen en el proceso de enseñanza y aprendizaje 
http://doi.org/10.15359/ree.25-2.16

Se puede concluir que las herramientas más utilizadas para mediar los procesos evaluativos son las herramientas web 2.0; la combinación de esta tecnología y una estrategia pedagógica pueden lograr desarrollar y evaluar la competencia esperada en el estudiantado (Williams et al., 2011). En la práctica, las competencias generan que haya un análisis mucho más profundo y claro de lo que se pretende evaluar, ya que al pensar en competencias se debe evidenciar no solo el concepto sino las aptitudes necesarias; básicamente lo que se genera con una competencia es: a) conocimiento y b) habilidad por medio de las rúbricas previamente establecidas (Summak et al., 2010). Adecuar una TIC a este tipo de estrategias conlleva a un nivel más alto de inclusión y de análisis, así llamada la tecnología instruccional o la inclusión de la tecnología educativa.

Fue posible considerar que las estrategias de evaluación utilizadas son de diferente índole, dependiendo del contexto en el que se desarrollan y su población de aplicación, según lo anterior, se pueden analizar algunas de estas estrategias tales como el aprendizaje autodirigido (Cheng et al., 2016), autorregulado (Navazesh et al., 2014) y centrado en el estudiantado (Summak et al., 2010). Estos tres aprendizajes, tomados como estrategias, son entendidos como la participación activa y principal del estudiantado mediante la manipulación de datos, análisis y una continua práctica, a través de un medio audiovisual que genere refuerzo cada cierto tiempo y evalúa constantemente.

Para citar algunas de las tecnologías que han sido utilizadas como estrategias, al ser las TIC una mediación y herramienta para la mejora de los procesos de evaluación, es posible hablar de los cursos web y presenciales (Ried, 2010), los cuales utilizan los materiales instruccionales como guías, libros, artículos y actividades interactivas, como instrumentos para fortalecer las temáticas trabajadas (Said et al., 2015).

Aunado a lo anterior, en estas estrategias y actividades, por medio de herramientas web, surgen materiales de tipo competencial, como los portafolios o e-portafolio (Wakimoto y Lewis, 2014), los cuales no solo ayudan al estudiantado a adquirir una responsabilidad por su aprendizaje, sino también a llevar un registro para su vida laboral. Además, recibe la ayuda de la prospectiva evaluativa, ya sea de tipo sumativa o formativa, del personal docente, pues ninguna de las dos intenciones de evaluación es errónea, sino depende de la integración que el personal docente quiera experimentar entre el uso de portafolio como estrategia y sobre el proceso de evaluación sumativo (Buente et al., 2015) y formativo (Cusi et al., 2013). Esto se ve, entonces, como un paso a la mejora de la evaluación.

Además de las estrategias y TIC que se han podido usar en las investigaciones revisadas, existe un punto importante entre lo que se quiere desarrollar y el resultado que se espera, aquí el tipo de evaluación ejerce un papel importante. Existen muchas combinaciones de evaluaciones, consecuentes con el tipo de ambiente de aprendizaje, ya sea presencial (Navazesh et al., 2014), e-learning (van Rooij, 2010), blended learning (Mathrani et al., 2016) y mobile learning (Ferenchick et al., 2013). 
http://doi.org/10.15359/ree.25-2.16

http://www.una.ac.cr/educare

educare@una.ac.cr

El principal hallazgo que se ha venido presentando a lo largo de todo el texto es la existencia de una correlación entre tipo de evaluación, la estrategia utilizada para desarrollar la clase y la TIC o tecnología que se utilice para mediar todo el proceso. Si se llega a esa intersección, se podría hablar de una coherencia en el proceso de enseñanza y aprendizaje que asegurará el éxito de lo planeado, tal como lo muestra la Figura 4.

Figura 4: Relación entre estrategias, evaluación y TIC

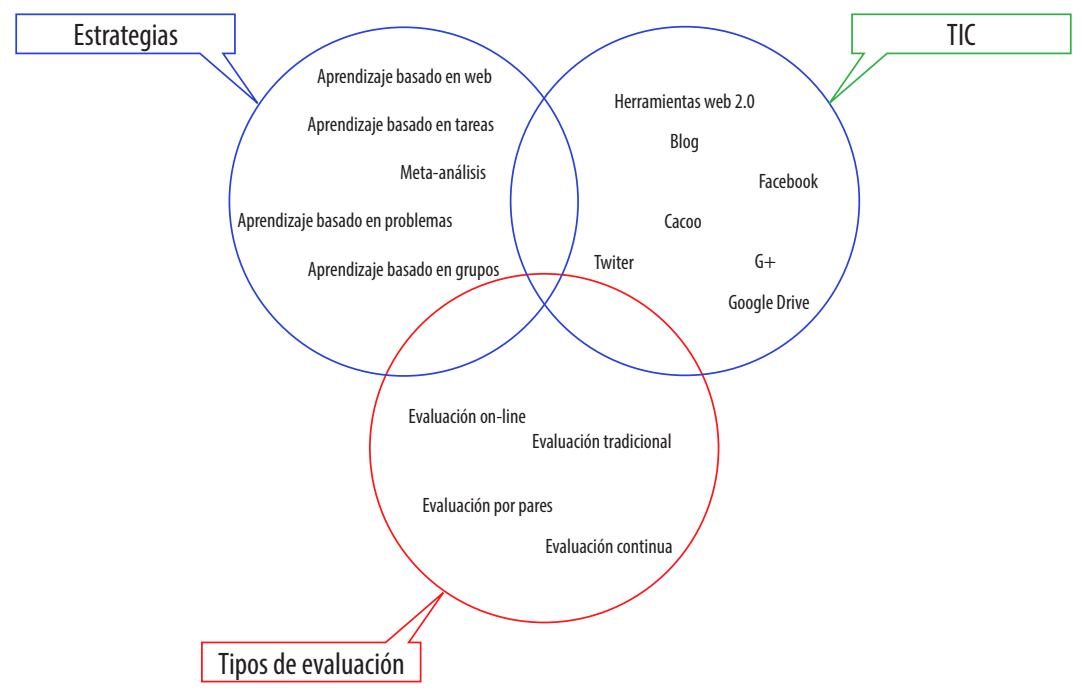

Nota: Elaboración propia.

Finalmente, existe un ítem de suma importancia, sin el cual la evaluación (estrategia, tipo de evaluación y TIC), no tendría sentido: la retroalimentación. La retroalimentación fue un término que se repetía continuamente en cada uno de los artículos de esta revisión, que alcanza a ser, de cierta manera, unificado pero distinguido, a través de sus características y el tiempo de aplicación. La retroalimentación es entendida como un aspecto crucial de la evaluación, de tal forma que se le entrega al estudiantado información relevante con la cual puede adaptar su aprendizaje después de un evento educativo (Cheng et al., 2016; Cusi et al., 2013; Ryan, 2013; van der Kleij et al., 2012).

Como se ha descrito previamente, se puede mencionar que la tecnología puede aportar bastante al complejo proceso de la evaluación, pues permite saber en qué intervalo de la práctica se debe sugerir un refuerzo y puede brindar un acompañamiento antes impensable por parte de un profesor o profesora, pues ahora lo podría hacer una máquina (Cusi et al., 2013), no obstante, es de necesaria acotación que, en muchas ocasiones, para determinar estos instantes, se utilizan las TIC como herramientas de ayuda para analizar los resultados y realizar un seguimiento al estudiantado, usando learning analytics (Wilson et al., 2016). 
http://doi.org/10.15359/ree.25-2.16

\section{Conclusiones}

Las estrategias encontradas en la presente revisión, entendidas como los métodos que son utilizados para realizar un proceso de evaluación, presentan mejoras respecto al cambio de la práctica del personal docente, la población de estudiantes a la que se le quiere aplicar el proceso, el contexto en la asignatura a intervenir $y$, finalmente, un cambio tecnológico en las herramientas y en el uso que se le da a las TIC.

Se concluye que aún predomina un uso de las TIC de manera instrumental, fundamentado en reemplazar el papel por una herramienta tecnológica. Como el caso de cuestionarios que siguen evaluando la apropiación de conceptos o teoría, pero que ahora lo hacen a través de alguna herramienta tecnológica. Ello supone una mejora al proceso, pero no es sustancial, aunque responde, de alguna manera, a la pregunta planteada en esta investigación.

Fue posible encontrar procesos de evaluación en donde el uso de las TIC se desarrolló de forma tal que generó un impacto sobre las asignaturas y temáticas intervenidas, sin convertir toda esta interacción en una distracción hacia el objetivo que se desea alcanzar con la evaluación, como el caso de juegos serios. En algunas ocasiones, el hecho de tener como herramienta una TIC generó una participación diferente hacia el concepto de evaluación, en donde tanto el personal docente como el estudiantado fueron partícipes de su propio proceso de aprendizaje; esto se evidenció en la evaluación continua y la evaluación por pares ayudada por los portafolios electrónicos, dentro de ambientes de aprendizajes presenciales.

Es claro que la tecnología abre muchas posibilidades al momento de evaluar, pero todo dependerá de cuatro elementos relevantes que se deben tener en cuenta. El contexto o el ambiente de aprendizaje en donde se realice el proceso educativo, la estrategia que conduzca la práctica de aula o pedagógica, los tipos de evaluación que se desean favorecer y que estén acordes con la estrategia planteada y, por último, las TIC o tecnologías que estén disponibles. Es evidente que muchas tecnologías usadas en el proceso de enseñanza y aprendizaje, como el caso de los portafolios, se vuelven la principal herramienta evaluativa; de esta misma manera sucede con los juegos serios, simuladores y otros tipos de recursos que, en sí mismos, realizan procesos de transmisión, evaluación, refuerzo y realimentación.

\section{Declaración de Material complementario}

Este artículo tiene disponible, como material complementario:

-La versión preprint del artículo en https://doi.org/10.5281/zenodo.3922916 
http://doi.org/10.15359/ree.25-2.16

http://www.una.ac.cr/educare

educare@una.ac.cr

\section{Referencias}

Amasha, M. yAlkhalaf, S. (2015). The effect of using facebook markup language (FBML) for designing an E-Learning Model in hgher education. International Journal of Research in Computer Science, 4(5), 1-9.

Ausubel, D. P. (1968). Educational psychology: A cognitive view. Holt, Rinehart and Winston.

Barry, S. (2012). A video recording and viewing protocol for student group presentations: Assisting self-assessment through a Wiki environment. Computers \& Education, 59(3), 85560. https://doi.org/10.1016/j.compedu.2012.04.008

Belland, B. R., Walker, A. E., Olsen, M. W. y Leary, H. (2015). A pilot meta-analysis of computerbased scaffolding in STEM education a pilot meta-analysis of computer-based scaffolding in STEM Education. International Forum of Educational Technology \& Society, 18(1), 183-97.

Bernard, R. M., Borokhovski, E., Schmid, R. F. y Tamim, R. M. (2014). An exploration of bias in meta-analysis: The case of technology integration research in higher education. Journal of Computing in Higher Education, 26(3), 183-209. https://doi.org/10.1007/s12528-0149084-z

Bordas Alsina, M. I. y Cabrera Rodríguez, F. Á. (2001). Estrategias de evaluación de los aprendizajes centrados en el proceso. Revista Española de Pedagogía, 59(218), 25-48. https://www.jstor. org/stable/23765840?seq=1

Buente, W., Winter, J. S., Kramer, H., Dalisay, F., Hill, Y. Z. y Buskirk, P. A. (2015). Program-Based Assessment of Capstone ePortfolios for a Communication BA Curriculum. International Journal of ePortfolio, 5(2), 169-179. http://theijep.com/pdf/IJEP191.pdf

Chapelle, C. A. y Voss, E. (2016). 20 years of technology and language assessment in Language Learning \& Technology. Language Learning \& Technology, 20(2), 116-128. https://www. Iltjournal.org/item/2950

Cheng, I. N. Y., Chan, J. K. Y., Kong, S. S. Y. y Leung, K. M. Y. (2016). Effectiveness and obstacle of using Facebook as a tool to facilitate student-centred learning in higher education. In AsiaPacific Forum on Science Learning and Teaching, 17(2), 2-10. https://www.eduhk.hk/apfslt/

Cohen, J. (1968). Weighted kappa: Nominal scale agreement provision for scaled disagreement or partial credit. Psychological bulletin, 70(4), 213-220. https://doi.org/10.1037/h0026256

Cusi, A., Morselli, F. y Sabena, C. (2013). The use of technology in formative assessment to raise achievement. FaSMEd. Position paper. https://research.ncl.ac.uk/fasmed/positionpapers/ The+use+of+technology+in+FA+to+raise+achievement Revision+UNITO-FINAL.pdf 
http://doi.org/10.15359/ree.25-2.16 http://www.una.ac.cr/educare educare@una.ac.cr

Dochy, F., Segers, M. y Dierick, S. (2002). Nuevas vías de aprendizaje y enseñanza y sus consecuencias: Una nueva era de evaluación. Revista de Docencia Universitaria, 2(2), 13-28. https://redined.mecd.gob.es/xmlui/handle/11162/91274?locale-attribute=gl

Esteve Mon, F. M. y Gisbert Cervera, M. (2011). El nuevo paradigma de aprendizaje y nuevas tecnologías. REDU. Revista de Docencia Universitaria, 9(3), 55-73. https://doi.org/10.4995/ redu.2011.6149

Ferenchick, G. S., Solomon, D., Foreback, J., Towfiq, B., Kavanaugh, K., Warbasse, L., Addison, J, Chames, F, Dandan, A. y Mohmand, A. (2013). Mobile technology for the facilitation of direct observation and assessment of student performance. Teaching and learning in medicine, 25(4), 292-299. https://doi.org/10.1080/10401334.2013.827972

Fink, A. (2013). Conducting research literature reviews: From the internet to paper. SAGE. https:// books.google.com.co/books?id=Dg5zAwAAQBAJ

García Laborda, J., Sampson, D. G., Hambleton, R. K. y Guzman, E. (2015). Guest editorial: Technology supported assessment in formal and informal learning. Educational Technology \& Society, 18(2), 1-2. https://www.j-ets.net/collection/published-issues/18_2

García-Valcárcel-Muñoz-Repiso, A., Basilotta-Gómez-Pablos, V. y López-García, C. (2014). Las TIC en el aprendizaje colaborativo en el aula de primaria y secundaria. Comunicar, 21(42), 6574. https://doi.org/10.3916/C42-2014-06

Gautreau, C. (2011). Motivational factors affecting the integration of a learning management system by faculty. Journal of Educators Online, 8(1), 1-23. https://eric.ed.gov/?id=EJ917870

Gómez-Escalonilla, M., Santín M. y Mathieu, G. (2011). La educación universitaria on-line en el periodismo desde la vision del estudiante. Comunicar, 19(37), 73-80. https://doi. org/10.3916/C37-2011-02-07

Gough, D., Thomas, J. y Oliver, S. (2012). Clarifying differences between review designs and methods. Systematic reviews, 1(28), 1-9. https://doi.org/10.1186/2046-4053-1-28

Hersh, M. (2014). Evaluation Framework for ICT-based learning technologies for disabled people. Computers \& Education, 78, 30-47. https://doi.org/10.1016/j.compedu.2014.05.001

$\mathrm{Hu}, \mathrm{C}$. (2016). Application of e-learning assessment based on AHP-BP algorithm in the cloud computing teaching platform. International Journal of Emerging Technologies in Learning (iJET), 11(08), 27-32. https://doi.org/10.3991/ijet.v11108.6039

Izquierdo, B. (2008). De la evaluación clásica a la evaluación pluralista: Criterios para clasificar los distintos tipos de evaluación. Empiria: Revista de Metodología de Ciencias Sociales, 16, 115-134. https://doi.org/10.5944/empiria.16.2008.1392 
http://doi.org/10.15359/ree.25-2.16

http://www.una.ac.cr/educare

educare@una.ac.cr

Jagodziński, P. y Wolski, R. (2015). Assessment of application technology of natural user interfaces in the creation of a virtual chemical laboratory. Journal of Science Education and Technology, 24(1), 16-28. https://doi.org/10.1007/s10956-014-9517-5

Kim, M. K., Zouaq, A. y Kim, S. M. (2016). Automatic detection of expert models: The exploration of expert modeling methods applicable to technology-based assessment and instruction. Computers \& Education, 101, 55-69. https://doi.org/10.1016/j.compedu.2016.05.007

Maas, N. A. y Flood, L. S. (2011). Implementing high-fidelity simulation in practical nursing education. Clinical Simulation in Nursing, 7(6), e229-e235. https://doi.org/10.1016/j. ecns.2010.04.001

Martín Gámez, C., Ruiz Mora, A. B. y Ruiz-Mora, I. (2015). El blog para la adquisición de competencias profesionales en la enseñanza superior. Revista Opcion, 31(Especial 2), 730752. https://www.redalyc.org/articulo.oa?id=31045568040

Mathrani, A., Christian, S. y Ponder-Sutton, A. (2016). PlaylT: Game based learning approach for teaching programming concepts. Educational Technology \& Society, 19(2), 5-17. https:// drive.google.com/file/d/1Z8tpXBV1hFX25QSpfG5Ih1la9LVFlrsh/view

Muñoz, J. M. (2008). NNTT, TIC, NTIC, TAC... en educación ¿pero esto qué es? Quaderns digitals: Revista de Nuevas Tecnologías y Sociedad, 51, 43-60. https://dialnet.unirioja.es/ ejemplar/186213

Navazesh, M., Rich, S. K. y Tiber, A. (2014). The rationale for and implementation of learnercentered education: Experiences at the Ostrow School of Dentistry of the University of Southern California. Journal of Dental Education, 78(2), 165-180.

Pásztor, A., Molnár, G.y Csapó, B. (2015). Technology-based assessment of creativity in educational context: The case of divergent thinking and its relation to mathematical achievement. Thinking Skills and Creativity, 18, 32-42. https://doi.org/10.1016/j.tsc.2015.05.004

Pirnay-Dummer, P., Ifenthaler, D. y Spector, J. M. (2010). Highly integrated model assessment technology and tools. Educational Technology Research and Development, 58(1), 3-18. https://doi.org/10.1007/s11423-009-9119-8

Ried, L. D. (2010). A distance education course in statistics. American Journal of Pharmaceutical Education, 74(9), 1-17. https://doi.org/10.5688/aj7409172

Ritchie,S.M.(2016).Self-assessment ofvideo-recorded presentations:Doesitimproveskills? Active Learning in Higher Education, 17(3), 207-221. https://doi.org/10.1177/1469787416654807 
Rust, C. (2002). The impact of assessment on student learning: How can the research literature practically help to inform the development of departmental assessment strategies and learner-centred assessment practices? Active Learning in Higher Education, 3(2), 145-158. https://doi.org/10.1177/1469787402003002004

Ryan, B. J. (2013). Line up, line up: Using technology to align and enhance peer learning and assessment in a student centred foundation organic chemistry module. Chemistry Education Research and Practice, 14(3), 229-238. https://doi.org/10.1039/C3RP20178C

Said, H., Kirgis, L., Verkamp, B. y Johnson, L. J. (2015). On-line vs. face-to-face delivery of information technology courses: Students' assessment. Journal of Information Technology Education: Research, 14, 297-312. https://doi.org/10.28945/2274

San Martín Cantero, D. (2014). Teoría fundamentada y Atlas. ti: Recursos metodológicos para la investigación educativa. Revista electrónica de investigación educativa, 16(1), 104-122. https://redie.uabc.mx/redie/article/view/727/906

Sánchez Vera, M. del M., Prendes Espinosa, M. P. y Fernández Breis, J. T. (2013). Tecnologías semánticas para la evaluación en red: Análisis de una experiencia con la herramienta OeLE. Revista de Investigación Educativa, 31(2), 447-464. https://doi.org/10.6018/rie.31.2.116721

Shih, R.-C. (2011). Can Web 2.0 technology assist college students in learning English writing? Integrating Facebook and peer assessment with blended learning. Australasian Journal of Educational Technology, 27(5), 829-845. https://doi.org/10.14742/ajet.934

Singh, L. (2013). Guided assessment or open discourse: A comparative analysis of students interaction on Facebook Groups. Turkish Online Journal of Distance Education, 14(1), 35-43. https://dergipark.org.tr/en/pub/tojde/issue/16895

Strauss, A. y Corbin, J. (2002). Bases de la investigación cualitativa. Técnicas y procedimientos para desarrollar la teoría fundamentada. Editorial Universidad de Antioquia.

Summak, M. S., Samancioğlu, M. y Bağlibel, M. (2010). Technology integration and assesment in educational settings. Procedia - Social and Behavioral Sciences, 2(2), 1725-1729. https://doi. org/10.1016/j.sbspro.2010.03.973

van der Kleij, F. M., Eggen, T. J. H. M., Timmers, C. F. y Veldkamp, B. P (2012). Effects of feedback in a computer-based assessment for learning. Computers \& Education, 58(1), 263-272. https:// doi.org/10.1016/j.compedu.2011.07.020

van Niekerk, E., Ankiewicz, P. y de Swardt, E. (2010). A process-based assessment framework for technology education: A case study. International Journal of Technology and Design 
http://doi.org/10.15359/ree.25-2.16

http://www.una.ac.cr/educare

educare@una.ac.cr

Education, 20(2), 191-215. https://doi.org/10.1007/s10798-008-9070-8

van Rooij, S. W. (2010). Higher Education and FOSS for e-Learning: The Role of Organizational Sub-cultures in Enterprise-wide Adoption. International Journal of Open Source Software and Processes (IJOSSP), 2(1), 15-31. https://doi.org/10.4018/jossp.2010010102

Wakimoto, D. K. y Lewis, R. E. (2014). Graduate student perceptions of eportfolios: Uses for reflection, development, and assessment. The Internet and Higher Education, 21, 53-58. https://doi.org/10.1016/j.iheduc.2014.01.002

Wang, S. (2014). The college physical education teaching evaluation based on the Fuzzy AHPEntropy and the computer simulation. International Journal of Multimedia and Ubiquitous Engineering, 9(10), 45-56. https://doi.org/10.14257/ijmue.2014.9.10.05

Wiliam, D. (2011). What is assessment for learning? Studies in Educational Evaluation, 37(1), 3-14. https://doi.org/10.1016/j.stueduc.2011.03.001

Williams, K., Wryobeck, J., Edinger, W., McGrady, A., Fors, U. y Zary, N. (2011). Assessment of competencies by use of virtual patient technology. Academic Psychiatry, 35(5), 328-330. https://doi.org/10.1176/appi.ap.35.5.328

Wilson, M., Gochyyev, P. y Scalise, K. (2016). Assessment of learning in digital interactive social networks: A learning analytics approach. Online Learning, 20(2), 97-119. https://doi. org/10.24059/olj.v20i2.799

Wu, J. (2011). Improving the writing of research papers: IMRAD and beyond. Landscape Ecology, 26(10), 1345-1349. https://doi.org/10.1007/s10980-011-9674-3

Yuretich, R. F. y Kanner, L. C. (2015). Examining the effectiveness of team-based learning (TBL) in different classroom settings. Journal of Geoscience education, 63(2), 147-156. https://doi. org/10.5408/13-109.1 